\title{
Neonatal jaundice causes and management
}

\author{
Ruya Althomali $^{1 *}$, Renad Aloqayli ${ }^{2}$, Basma Alyafi ${ }^{3}$, Ahela Nono ${ }^{3}$, Suhaib Alkhalaf ${ }^{4}$, Abdulaziz \\ Aljomailan $^{4}$, Hesham AlHarbi ${ }^{5}$, Abdulrahman Alqahtani ${ }^{6}$, Hawra Alherz ${ }^{7}$, Moluk Aldebani ${ }^{3}$
}

\author{
${ }^{1}$ Taif University, Taif, KSA \\ ${ }^{2}$ Unaizah College of Medicine, Unaizah, KSA \\ ${ }^{3}$ Ibn Sina National College for Medical Studies, Jeddah, KSA \\ ${ }^{4}$ Qassim University, Qassim, KSA \\ ${ }^{5}$ Cairo University, Cario, Egypt \\ ${ }^{6}$ King Khalid University, Abha, KSA \\ ${ }^{7}$ Primary Health Care Ministry of Health, Dammam, KSA
}

Received: 02 October 2018

Accepted: 18 October 2018

\section{*Correspondence: \\ Dr. Ruya Althomali, \\ E-mail: ro2ya@live.com}

Copyright: ( $\odot$ the author(s), publisher and licensee Medip Academy. This is an open-access article distributed under the terms of the Creative Commons Attribution Non-Commercial License, which permits unrestricted non-commercial use, distribution, and reproduction in any medium, provided the original work is properly cited.

\begin{abstract}
$80 \%$ of healthy neonates present with some degree of hyperbilirubinemia after birth, however, only 5-10\% would require therapy to prevent damage or treat the cause of jaundice. Neonatal jaundice can be classified as physiological and pathological and can have several causes such as breast milk feeding, blood group incompatibility, hemolysis, or genetic defects of enzymes in the bilirubin metabolism pathway. We tried to understand the various types of neonatal jaundice, and also focus on its management. We conducted this review using a comprehensive search of MEDLINE, PubMed and EMBASE from January 2001 to March 2017. The following search terms were used: neonatal jaundice, hyperbilirubinemia, ABO incompatibility, neonatal hemolysis, kernicterus, phototherapy, exchange transfusion. Hyperbilirubinemia and jaundice are common issues encountered neonates and infants. Most cases of neonatal hyperbilirubinemia and jaundice are physiological and benign. However, some severe cases may progress to develop severe and permanent long-term complications. Therefore, early diagnosis and management is essential. Neonatal jaundice can be treated using phototherapy, pharmacological agents, intravenous immunoglobulins and exchange transfusion in severe cases.
\end{abstract}

Keywords: Neonatal jaundice, Neonatal hyperbilirubinemia, Prematurity, Kernicterus

\section{INTRODUCTION}

It has been reported that up to $80 \%$ of healthy neonates can present with idiopathic jaundice at birth. Neonatal jaundice can be defined as the presence of yellowish color of the sclera and the skin in a neonate, caused by bilirubin accumulation. Neonatal jaundice will immediate cause parents to be anxious, and physicians to be concerned of its causes. The National Neonatal-Perinatal Database reports the incidence of hyperbilirubinemia in neonates to be $3.3 \%$ in live births in house. On the other hand, hyperbilirubinemia-related morbidity in extramural admissions can reach $22.1 \%$. When bilirubin levels increase in a neonate, the first thing to notice is yellowish discoloration of the dermal icterus, which will later continue to include the extremities and the body. This process is usually physiologic and can occur in more than half of the neonates during their first week of life. ${ }^{1}$

However, significant increases in bilirubin levels are usually toxic and cause significant damage to the development of the central nervous system. They may also be associated with neurological and behavioral impairments on both preterm and full-term neonates. 
Therefore, management of hyperbilirubinemia will be required in about $5-10 \%$ of neonates with jaundice in order to prevent these consequences. ${ }^{2}$

Predisposing factors and risk factors associated with the development of neonatal jaundice are many and include: genetic predisposition, racial disparities, inherited defects, Gilbert's syndrome, Crigler Najjar 1 and 2, and spherocytosis. Many previous studies have suggested the strong association between neonatal hyperbilirubinemia, and certain genetic variations that alter the activity of enzymes. Examples of these include a variation in the gene encoding for the the uridine 5'-diphosphate glucuronosyltransferase 1A1 enzyme, which will lead to a significant reduction in the activity of the gene in both children and adults, leading to the accumulation of unconjugated bilirubin. Another example is the variation in the gene encoding for the organic anion transporter 2 (OATP2) which leads in severe neonatal hyperbilirubinemia. ${ }^{3}$

\section{METHODS}

\section{Data sources and search terms}

We conducted this review using a comprehensive search of MEDLINE, PubMed and EMBASE, from January 2001 to March 2017. The following search terms were used: neonatal jaundice, hyperbilirubinemia, ABO incompatibility, neonatal hemolysis, kernicterus, phototherapy, exchange transfusion.

\section{Data extraction}

Two reviewers have independently reviewed the studies, abstracted data and disagreements were resolved by consensus. Studies were evaluated for quality and a review protocol was followed throughout.

\section{TYPES OF JAUNDICE}

\section{Physiological jaundice}

Most cases of hyperbilirubinemia in neonates are in fact physiologic and lead to no serious complications. In rare cases of physiologic hyperbilirubinemia, where bilirubin levels reach toxic high levels, neurodevelopmental abnormalities could occur including intellectual deficits, athetosis, and loss of hearing. Physiological jaundice usually appears after at least 24 hours of birth, and peak after four or five days. It later disappears after about 2 weeks of life. In physiologic jaundice, bilirubin is predominantly unconjugated, and bilirubin levels in serum do not become higher than $15 \mathrm{mg} / \mathrm{dl}$. More recent guidelines have suggested that even bilirubin levels that reach 17 or $18 \mathrm{mg} / \mathrm{dl}$ could be considered normal and physiological in an otherwise healthy full-term neonate. ${ }^{4,5}$

\section{Breast feeding/milk jaundice}

Neonates and infants who are exclusively breastfed can develop other types of jaundice related to breastfeeding. Similar to physiological jaundice, breast feeding jaundice appears after a day or two days of birth, and peaks at the first week of age. However, it can last longer than physiological jaundice, and usually will not completely disappear until the third of fourth week of life. Moreover, infants with breast feeding jaundice have been found to have higher levels of bilirubin than physiological jaundice. Moreover, in some infants, breast feeding jaundice can be recurrent as long as the breastfeeding period continues. It is rare for breast feeding jaundice to lead to the accumulation of toxic levels of bilirubin. In these cases, serious cerebral lesions can occur leading to the development of what is called nuclear jaundice, which could manifest with mental retardation, behavioral disorders, and hearing loss. ${ }^{6}$

It is estimated that up to $30 \%$ of breastfed infants will develop a mild jaundice within the first three weeks of their life. This jaundice could sometimes persist for up to three months. On the other hand, the reduction of breastfeeding frequency will increase the risk and severity of physiological jaundice. Therefore, the best management of this jaundice in an otherwise healthy fullterm baby is to encourage mothers to continue breastfeeding for at least ten times daily. ${ }^{7}$

The breast milk of the mother can also affect the occurrence of hyperbilirubinemia in a neonate. In fact, up to $4 \%$ of infants who are exclusively breastfed can develop jaundice within the first three weeks of life, where bilirubin levels can become higher than $10 \mathrm{mg} / \mathrm{dl}$. In these cases, if the jaundice persists for longer time, it should be managed as prolonged jaundice. In breast milk jaundice, bilirubin is usually unconjugated, which could confuse this case with other cases, and make it important to exclude more serious etiologies. It is important to encourage mothers to increase the frequency of breastfeeding until bilirubin levels return to their normal values. However, if bilirubin levels are higher than 20 $\mathrm{mg} / \mathrm{dl}$ it is recommended to stop breastfeeding, as this could be associated with permanent neurological complications. $^{7}$

\section{Hemolytic jaundice}

\section{1) Rh factor hemolytic disease}

In cases where alloimmunization of maternal RBCs, Rhesus hemolytic disease of the newborns (RHDN) occurs. In these cases, the mother's body produces antibodies that attack specific antigens on the RBCs of the fetus, most likely, the Rh antigens. This is most likely to occur when an Rh negative mother has an Rh positive fetus. In these cases, the mother produces antibodies against the $\mathrm{Rh}$ antigen, and these antibodies can cross the placenta and enter the circulation of the fetus. This will 
cause the fetus to develop a clinical picture that ranges from mild hemolytic anemia, to severe hemolytic anemia followed by fetal hydrops. Infants who have a risk of developing Rhesus hemolytic disease of the newborns should be early investigated and treated. Investigations for these infants include packed cell volume, blood group, Rh typing, serum bilirubin, and reticulocytes count. Immediately following birth, these patients should be initiated on phototherapy, which should continue until the level of bilirubin decreases significantly. ${ }^{8}$

\section{2) $A B O$ incompatibility}

It is estimated that $\mathrm{ABO}$ incompatibility between the fetus and the mother can occur in up to $20 \%$ of pregnancies, and results from an $\mathrm{O}$ mother and who has an A or B fetus. Therefore, it is important to closely monitor mothers who have an O blood group for at least three days after birth. However, it is not generally recommended to perform routine screening of cord blood in their infants. ABO incompatibility jaundice usually starts to appear at the age of one day. If it appears earlier, or it becomes severe, further investigations must be done to rule out more severe etiologies. Intensive phototherapy is indicated in these cases. ${ }^{9}$

\section{3) G6PD deficiency}

The management of spherocytosis, G6PD deficiency, and similar diseases is similar to the management of $\mathrm{ABO}$ incompatibility. Of these, the most common one is G6PD deficiency. It causes significant dysfunction of the hexose monophosphate pathway within the RBCs leading to hemolysis. It should be suspected in any neonate who has a family history with hemolytic jaundice, or who originates from are where G6PD is prevalent. ${ }^{10}$

\section{CLINICAL EVALUATION}

Kramer was the first to suggest the use of dermal staining to clinically guide physicians and help them determine severity of jaundice. The progression of dermal straining occurs in a direction that is cephalo-caudal. It is important to examine infants in good light conditions. Moreover, the examination should include paling the neonate's skin with pressure to be able to note the underlying skin and subcutaneous color. Urgent laboratory investigations, including bilirubin levels, must be collected in any infant with yellow skin that extends beyond the thighs. In infants who have already received phototherapy, or who have dark skin, clinical examination may not provide reliable assessment of the condition. $^{11}$

\section{Assessment of bilirubin}

The serum bilirubin level is usually checked using biochemical methods, transcutaneous bilirubinometer or Bilimeter. $^{12}$

\section{Biomechanical}

The best method for the assessment of bilirubin levels is the measurement of both conjugated and total bilirubin using the reaction of van den Bergh. ${ }^{12}$

\section{Bilimeter}

Bilimeter is used to measure the levels of total bilirubin in the serum and is based on spectrophotometry techniques. This is a general method to use in neonates due to the predominance of unconjugated bilirubin. ${ }^{12}$

\section{Transcutaneous bilirubinometer}

This is a noninvasive method that uses the spectral reflectance of multi wavelengths that are released from the skin bilirubin. This instrument's accuracy is usually affected by the pigmentation of the skin and its thickness. $^{13}$

\section{CLINICAL APPROACH}

The first step in the evaluation of an infant who has jaundice is to distinguish between pathological jaundice and physiological jaundice. The AAP has released their guidelines in this regard as the following. ${ }^{14}$

\section{Evidence of hemolysis}

When the onset starts within the first day of life, this could immediately raise the suspicion for pathological jaundice, most likely hemolytic. Other alarming presentations include pallor, hydrops fetalis, hepatosplenomegaly, hemolysis appearing on blood smear, reticulocytosis, rapid elevation in bilirubin levels, and positive family history for jaundice or hemolysis. ${ }^{9}$

\section{Instructions and precautionary measure for parents during physiological jaundice}

Jaundice usually has a benign course, which should be clearly explained to parents. It is essential to encourage the mother to exclusively breastfeed her neonate more frequently, and for more than eight times daily, with no other feeding or water. It is also important to inform parents that they should keep monitoring their child and call the doctor immediately of the jaundice progresses to the legs. Finally, any infant must be reevaluated at the age of four days, to make sure breastfeeding is sufficient, and rule out the presence of pathologies. ${ }^{15}$

\section{Management of pathological jaundice}

It is recommended to perform laboratory investigations to assess bilirubin levels in the serum of any infant with jaundice. Management and treatment should be started when there is pathological jaundice. Generally, if the jaundice starts within less than 24 hours following birth, it is considered pathological and managed as a hemolytic 
jaundice. All these investigations must be acquired: blood group, RH type, DCT, packed cell volume, blood smear, RBCs morphology, reticulocytes count, and G6PD enzyme levels. These investigations will diagnose most causes and etiologies of hemolytic jaundice. Phototherapy should be started in these cases and continued until the bilirubin level sufficiently decreases. When there is any suspicion that there might be encephalopathy and/or kernicterus, exchange transfusion must be immediately started regardless of the levels of bilirubin in the serum. ${ }^{15}$

\section{TREATMENT OPTIONS FOR JAUNDICE}

Treatment of jaundice is grouped into two main categories: phototherapy and pharmacological therapy. Phototherapy is further classified into conventional, intensive, and exchange transfusion, whereas pharmacological therapy is further grouped into IV immunoglobulins, phenobarbitone, and metalloporphyrins. ${ }^{16}$

\section{Phototherapy}

Phototherapy provides an easy and safe method to treat hyperbilirubinemia with minimal side effects. Its efficacy mainly depends on the exposure to the phototherapy; for example, single surface phototherapy is significantly less effective than double surface phototherapy. ${ }^{17}$ Moreover, the use of phototherapy continuously is associated with better outcomes than its use intermittently. It is recommended not to interrupt phototherapy except during breastfeeding. Type of phototherapy are as follows. ${ }^{14,18}$

\section{Conventional phototherapy}

This is used in mild and non-hemolytic neonatal jaundice.

\section{Intensive phototherapy}

This is use in more severe cases, severe elevations in bilirubin, hemolytic jaundice, or failure of the conventional phototherapy to relieve the jaundice.

\section{Exchange transfusion}

This is used to remove antibodies that are causing hemolysis and is used in Rh Isoimmunization and ABO Incompatibility.

\section{Pharmacological treatment}

Pharmacological therapy of jaundice is usually grouped into the following categories:

\section{Phenobarbitone}

This agent work by decreasing the processing of bilirubin, that includes the uptake, conjugation, and excretion of the bilirubin by the liver. This will eventually lead to a significant reduction in the levels of bilirubin in the blood. However, phenobarbitone has a slow onset, and takes a few days to effectively work. It has been found to lead to a significant improvement of jaundice in neonates with hemolytic jaundice following three to five days of treatment. It also has a relatively safe profile, with minimal adverse events. The use of phenobarbitone in the management and treatment of neonatal jaundice is supported by solid evidence and large studies. ${ }^{19}$

\section{Intravenous immunoglobulin (IVIG)}

Several studies have demonstrated the efficacy of high doses of IVIG in the treatment of neonatal jaundice. ${ }^{20}$

\section{Metalloporphyrins}

These drugs are still in the experimental phases with no strong evidence supporting their use. However, results are promising for the management and treatment of both hemolytic and nonhemolytic jaundice in the neonates. Moreover, they have been shown to be associated with relatively safe profiles and minimal adverse events. ${ }^{21}$

\section{FOLLOW-UP}

It is essential to strictly monitor and follow up infants who develop jaundice, especially if their bilirubin levels exceeded $20 \mathrm{mg} / \mathrm{dl}$ or they required exchange transfusion. This is due to their higher risk of developing permanent neurological disorders. In addition, all these infants must be assessed for hearing loss using a Brainstem Evoked Response Audiometry at the age of three months. ${ }^{22}$

\section{CONCLUSION}

Hyperbilirubinemia and jaundice are common issues encountered neonates and infants. Most cases of neonatal hyperbilirubinemia and jaundice are physiological and benign. However, some severe cases may progress to develop severe and permanent long-term complications. Therefore, it is essential to early diagnose and manage pathological causes of neonatal jaundice. Most causes of pathological jaundice are due to hemolysis. Neonatal jaundice can be mainly treated using phototherapy. Other pharmacological therapies include phenobarbitone and IV immunoglobulins. Exchange transfusion is used in severe cases or when other measures fail to improve the jaundice.

\section{Funding: No funding sources \\ Conflict of interest: None declared \\ Ethical approval: Not required}

\section{REFERENCES}

1. Narional Neonatal-Perinatal Databaase. NNPD Network, Indian Council of Medical Research, New Delhi. 2003; Available at: http://www.newbornw 
hocc.org/pdf/nnpd_report_2002-03.PDF. Accessed on 3 June 2018.

2. Chou SC, Palmer RH, Ezhuthachan S, Newman C, Pradell-Boyd B, Maisels MJ, et al. Management of hyperbilirubinemia in newborns: measuring performance by using a benchmarking model. Pediatrics. 2003;112:1264-73.

3. Huang MJ, Kua KE, Teng HC, Tang KS, Weng HW, Huang CS. Risk factors for severe hyperbilirubinemia in neonates. Pediatr Res. 2004;56:682-9.

4. Clarkson JE, Cowan JO, Herbison GP. Jaundice in full term healthy neonates--a population study. Aust Paediatr J. 1984;20:303-8.

5. Dennery PA, Seidman DS, Stevenson DK. Neonatal hyperbilirubinemia. N Engl J Med. 2001;344:58190.

6. Gartner LM, Lee KS. Jaundice in the breastfed infant. Clin Perinatol. 1999;26:431-45.

7. Schneider AP. Breast milk jaundice in the newborn. A real entity. JAMA. 1986;255:3270-74.

8. Stockman JA. Overview of the state of the art of $\mathrm{Rh}$ disease: history, current clinical management, and recent progress. J Pediatr Hematol Oncol. 2001;23:554-62.

9. Murray NA, Roberts IA. Haemolytic disease of the newborn. Arch Dis Child Fetal Neonatal Ed. 2007;92:83-8.

10. Kaplan M, Hammerman C. Severe neonatal hyperbilirubinemia. A potential complication of glucose-6-phosphate dehydrogenase deficiency. Clin Perinatol. 1998;25:575-90.

11. Johnson L, Bhutani VK. Guidelines for management of the jaundiced term and near-term infant. Clin Perinatol. 1998;25:555-74.

12. Watson D, Rogers JA. A study of six representative methods of plasma bilirubin analysis. J Clin Pathol. 1961;14:271-8.

13. Bosschaart N, Kok JH, Newsum AM, Ouweneel DM, Mentink R, van Leeuwen TG, et al. Limitations and opportunities of transcutaneous bilirubin measurements. Pediatrics. 2012;129:68994.

14. American Academy of Pediatrics Subcommittee on H. Management of hyperbilirubinemia in the newborn infant 35 or more weeks of gestation. Pediatrics. 2004;114:297-316.

15. Maisels MJ, Watchko JF, Bhutani VK, Stevenson DK. An approach to the management of hyperbilirubinemia in the preterm infant less than 35 weeks of gestation. J Perinatol. 2012;32:660-4.

16. Ennever JF. Blue light, green light, white light, more light: treatment of neonatal jaundice. Clin Perinatol. 1990;17:467-81.

17. Cremer RJ, Perryman PW, Richards DH. Influence of light on the hyperbilirubinaemia of infants. Lancet. 1958;1:1094-7.

18. Maisels MJ. Phototherapy--traditional and nontraditional. J Perinatol. 2001;21:93-97;104-107.

19. Shankaran S, Woldt E, Nelson J, Bedard M, Delaney-Black V. Antenatal phenobarbital therapy and neonatal outcome. II:Neurodevelopmental outcome at 36 months. Pediatrics. 1996;97:649-52.

20. Alpay F, Sarici SU, Okutan V, Erdem G, Ozcan O, Gokcay E. High-dose intravenous immunoglobulin therapy in neonatal immune haemolytic jaundice. Acta Paediatr. 1999;88:216-9.

21. Vreman HJ, Ekstrand BC, Stevenson DK. Selection of metalloporphyrin heme oxygenase inhibitors based on potency and photoreactivity. Pediatr Res. 1993;33:195-200.

22. Facchini FP, Mezzacappa MA, Rosa IR, Mezzacappa Filho F, Aranha-Netto A, Marba ST. Follow-up of neonatal jaundice in term and late premature newborns. J Pediatr (Rio J). 2007;83:31322.

Cite this article as: Althomali R, Aloqayli R, Alyafi B, Nono A, Alkhalaf S, Aljomailan A, et al. Neonatal jaundice causes and management. Int J Community Med Public Health 2018;5:4992-6. 